\title{
Accelerated Middle Half Body Radiotherapy in Bone Metastases from Prostate Cancer: A Phase I Study (SHARON Project)
}

\author{
ALICE ZAMAGNI ${ }^{1}$, MILLY BUWENGE ${ }^{1}$, GABRIELLA MACCHIA ${ }^{2}$, GIAMBATTISTA SIEPE ${ }^{1}$, \\ SAVINO CILLA ${ }^{3}$, FRANCESCO CELLINI ${ }^{4}$, ALESSANDRA ARCELLI ${ }^{1}$, ELEONORA FARINA $^{5}$, \\ FRANCESCO DEODATO $^{2}$, SILVIA CAMMELLI ${ }^{1 *}$ and ALESSIO G. MORGANTI ${ }^{1 *}$ \\ ${ }^{1}$ Department of Experimental, Diagnostic and Specialty Medicine - DIMES, \\ University of Bologna, S. Orsola-Malpighi Hospital, Bologna, Italy; \\ ${ }^{2}$ Radiation Oncology Unit, Research and Care Foundation 'Giovanni Paolo II', \\ Catholic University of Sacred Heart, Campobasso, Italy; \\ ${ }^{3}$ Medical Physics Unit, Research and Care Foundation 'Giovanni Paolo II', \\ Catholic University of Sacred Heart, Campobasso, Italy; \\ ${ }^{4}$ Fondazione Policlinico Universitario "A. Gemelli" IRCCS, UOC Radioterapia Oncologica, \\ Dipartimento di Diagnostica per Immagini, Radioterapia Oncologica ed Ematologia, Rome, Italy; \\ ${ }^{5}$ Radiation Oncology Unit, Maria Cecilia Hospital, GVM Care \& Research, Cotignola, Italy
}

\begin{abstract}
Background/Aim: The primary endpoint of this phase I study was the maximum tolerated dose (MTD) of middle half body $(M H B)$ accelerated radiotherapy $(R T)$ in multiple bone metastatic (BM) prostate cancer (PCa) patients. Patients and Methods: Three step dose escalation [13 Gy (3.25 Gy/fraction), 14 Gy (3.5 Gy/fraction), and 15 Gy (3.75 Gy/fraction)] in three consecutive patient cohorts were planned. RT was delivered in two consecutive days and two daily fractions. Six patients were enrolled in the first two cohorts and 12 in the third cohort. Grade $\geq 3$ toxicity was considered as a dose-limiting toxicity (DLT). Results: Twenty-five patients (median age $=71$ years, median followup=7.4 months) were enrolled. Defined MTD dose was 15 Gy. Overall pain response rate was 76\%: 9 patients (36\%) showed complete and 10 patients (40\%) reported partial response of pain. Conclusion: MHB accelerated RT (total dose: 15 Gy) delivered in two consecutive days and two daily fractions is well tolerated.
\end{abstract}

\footnotetext{
*These Authors contributed equally to this study.

Correspondence to: Milly Buwenge, MSc, Radiation Oncology Center, Department of Experimental, Diagnostic and Specialty Medicine - DIMES, University of Bologna, S. Orsola-Malpighi Hospital, via Giuseppe Massarenti 9, 40138 Bologna, Italy. Tel: +39 0512143564, Fax: +39 0516364336, e-mail: mbuwenge@gmail.com
}

Key Words: Prostate neoplasm, bone metastases, radiotherapy, palliative therapy, phase I.
Worldwide, prostatic adenocarcinoma (PCa) represents the second most common cancer in men and the 6th cause of cancer-related death (1). In about $80 \%$ of PCa patients, the initial and main site of metastasis is the skeleton (2). Twoand 5-year overall survival (OS) in PCa patients with bone metastases (BM) at the time of diagnosis are $90 \%$ and $36 \%$, respectively, (median $\mathrm{OS}=42.1$ months) (3) representing higher rates when compared to patients with visceral metastases (4).

Palliative radiotherapy (RT) is a treatment option in BM (5) to reduce pain, prevent pathological fractures, and to improve quality of life (QoL). However, patients requiring palliative RT may suffer from physical discomfort due to transfers to the RT center and/or toxicity, and from emotional distress particularly in case of prolonged treatment duration. These factors can partially explain why in the USA only $3 \%$ of hospice patients are treated with RT (6). Based on this background, an optimization of palliative RT is needed with both the aims of reducing toxicity and treatment duration, ultimately improving QoL.

In the past decades, several studies reported effective and rapid palliation after half-body $\mathrm{RT}$ on multiple BM in patients with different primary tumors $(7,8)$. In the 90's, Salazar and colleagues evaluated different half-body irradiation treatment schedules (9) and in 2001 the outcomes from a multicenter randomized study sponsored by the International Atomic Energy Agency were published (10). The delivery of 12 Gy in two consecutive days with two fractions/day resulted in an effective and tolerable dose, similar to 15 Gy in 5 daily fractions regimen in several metastatic malignancies but was 
less effective in PCa patients. In fact, an improved outcome with lower toxicity was observed in PCa patients receiving the prolonged treatment (15 Gy) (10).

Our study aimed to analyze the feasibility to deliver the same dose (15 Gy) in just two days (two fractions/day) instead of five in $\mathrm{PCa}$ patients with $\mathrm{BM}$, in this way combining the advantages of higher dose and shorter duration. All patients were irradiated in the site with the highest incidence of BM from PCa, i.e. in the Middle Half Body (MHB) including the lumbar spine, pelvic bones, and femurs. To reduce the irradiation of the organs at risk, RT was delivered with a multiple beams 3D-conformal technique.

\section{Patients and Methods}

Endpoints and study design. We planned a dose escalation trial having as primary endpoint the definition of the maximum tolerated dose (MTD) of an accelerated MHB RT regimen in the setting of $\mathrm{PCa}$ BM. Secondary endpoint was symptomatic response (complete/partial reduction of pain). The dose level lower than that associated with an incidence of dose limiting toxicity (DLT) of at least $1 / 3$ of the patients was considered as the MTD (11). Any $G \geq 3$ acute toxicity according to the Radiation Therapy Oncology Group (RTOG) scale (12), was considered as DLT.

We planned to enroll at least six patients in the first two patient cohorts (dose level 1 and 2) while we decided to enroll at least 12 patients in the third cohort (dose level 3: $15 \mathrm{~Gy}$ ) in order to achieve a more precise estimate of the toxicity rate produced by the highest dose. In the first two cohorts, we planned to start the enrolment in the subsequent dose-level only in case of $<2 / 6$ recorded DLT and provided the sixth patient was observed for at least 3 months. In the same two cohorts, in case of expansion to 12 patients, we planned to proceed with enrollment to the next dose level if $<4 / 12$ patients had DLT. If 2/6 recorded DLT in a single cohort, we planned for an additional enrollment in the same cohort of six patients. Furthermore, we scheduled the trial to be interrupted for $>2 / 6$ or $\geq 4 / 12$ patients with DLT at a given dose level. In this case, the MTD was considered as the dose level lower than the latter.

Eligibility criteria. We used the following inclusion criteria: biopsy proven $\mathrm{PCa}$, painful BM localized in pelvic bones, femurs, and lumbar spine, age $\geq 18$ years, Eastern Cooperative Oncology Group (ECOG) $\leq 3$, life expectancy $>3$ months, adequate bone marrow function defined as: hemoglobin $>8 \mathrm{~g} / \mathrm{dl}$, neutrophils $>1500 / \mathrm{mcl}$, platelets $>100.000 / \mathrm{mcl}$. Furthermore, only castration resistant PCa patients were enrolled to avoid any confounding effect on symptomatic response produced by androgen deprivation therapy. Castration-resistant patients were defined as patients with progressive $\mathrm{PCa}$ (biochemical or clinical progression) and testosterone castrate levels.

We evaluated all patients before enrollment, with whole body computed tomography (CT) scan, bone scintigraphy, complete medical history, physical examination, hematocrit test, platelet count, and differential white blood cell count. Before and after treatment, at each control visit, information on pain status, ECOG, and QoL was registered. Pain scoring was performed using the Visual Analogue Score (VAS) (13). Furthermore, we used the International Atomic Energy Agency Pain- and Drug-Scores to grade the intensity of pain and analgesics use (10).
Table I. Clinical target volume (CTV) modulation.

\begin{tabular}{ll}
\hline Clinical target volumes & Number of patients $(\%)$ \\
\hline D12-L5, bony pelvis, 1/3 proximal femurs & $2(8)$ \\
L2-L5, bony pelvis, 1/3 proximal femurs & $2(8)$ \\
L3-L5, bony pelvis, 1/3 proximal femurs & $2(8)$ \\
D12-L5, bony pelvis, entire femurs & $2(8)$ \\
L1-L5, bony pelvis, entire femurs & $2(8)$ \\
L2-L5, bony pelvis, entire femurs & $3(12)$ \\
L3-L5, bony pelvis, entire femurs & $9(36)$ \\
L4-L5, bony pelvis, entire femurs & $3(12)$ \\
\hline
\end{tabular}

We performed QoL evaluation with the Cancer Linear Analogue Scales (CLAS), considering comfort (CLAS1), energy level (CLAS2), and ability to perform normal daily tasks (CLAS3) (14).

Treatment. The treatment was planned using CT-simulation in a supine position from the level of the T12 vertebra to the upper end of the tibia, with a distance between slices of $5 \mathrm{~mm}$. The clinical target volume (CTV) was modulated depending on BM localization to include the metastatic lumbar vertebrae and the immediately superior vertebra, all the bony structures of the pelvis, and the entire femurs if involved below the proximal epiphysis. Instead, only the proximal third of the femurs was included in the CTV in the case of metastases localized only at the level of the femoral head. The planning target volume (PTV) was defined by adding a $1-\mathrm{cm}$ isotropic margin to the CTV.

Patients received 13 Gy (3.25 Gy per fraction), 14 Gy (3.5 Gy per fraction), or 15 Gy (3.75 Gy per fraction) in the three subsequent patient cohorts. We estimated the probability of late toxicity (using an $\alpha / \beta$ ratio of 3 ) by calculating the Equivalent Dose in $2 \mathrm{~Gy} /$ fractions $\left(\mathrm{EQD}_{2}\right)$. The values of $\mathrm{EQD}_{2}$ were $16.2,18.2$, and $20.2 \mathrm{~Gy}$ for the 3 dose levels, respectively (15). Dose specification was performed based on the ICRU Report 62 (16). RT was delivered using $3 \mathrm{D}$-conformal four-field (box) technique in two consecutive days and two daily fractions with at least 6-h interval.

The quality of the treatment was assured with independent checks by medical and physical personnel both before and during the treatment (17). The accuracy of the set-up was evaluated before the delivery of each fraction through the use of an EPID system (18).

No patient received a new systemic therapy (compared to the previous hormonal therapy) before symptomatic or clinical progression.

Follow-up evaluation. Fifteen days after the end of RT we performed the first follow-up visit evaluating the full blood count, type and grade of acute toxicity, pain control, and QoL. Subsequently, patients were evaluated every 2-months to score late toxicity, pain control, and QoL.

Grading of acute and late toxicities was performed using the RTOG and RTOG/European Organization for Research and Treatment of Cancer (RTOG/EORTC) scores, respectively (12). Pain and drug score and QoL indices (CLAS1, CLAS2, CLAS3) were recorded at each visit.

The evaluation of pain response was performed based on the International Consensus on Palliative Radiotherapy criteria (19) as 
Table II. Acute toxicity (data presented as number of patients, with percentages in parenthesis).

\begin{tabular}{|c|c|c|c|c|c|c|c|}
\hline & \multirow[b]{2}{*}{ Grade } & \multicolumn{6}{|c|}{ Dose level } \\
\hline & & \multicolumn{2}{|c|}{1} & \multicolumn{2}{|c|}{2} & \multicolumn{2}{|c|}{3} \\
\hline Enrolled patients & & \multicolumn{2}{|c|}{6} & \multicolumn{2}{|c|}{7} & \multicolumn{2}{|c|}{12} \\
\hline \multicolumn{8}{|l|}{ Acute toxicity } \\
\hline Skin & 1 & 1 & $(16.7 \%)$ & 0 & $(0.0 \%)$ & 0 & $(0.0 \%)$ \\
\hline Lower gastrointestinal tract & 1 & 0 & $(0.0 \%)$ & 0 & $(0.0 \%)$ & 1 & $(8.3 \%)$ \\
\hline \multirow{2}{*}{ Upper gastrointestinal tract } & 1 & 0 & $(0.0 \%)$ & 1 & $(14.3 \%)$ & 2 & $(16.7 \%)$ \\
\hline & 2 & 0 & $(0.0 \%)$ & 0 & $(0.0 \%)$ & 2 & $(16.7 \%)$ \\
\hline White blood cell count & 1 & 0 & $(0.0 \%)$ & 0 & $(0.0 \%)$ & 1 & $(8.3 \%)$ \\
\hline \multirow[t]{2}{*}{ Flare up } & 1 & 0 & $(0.0 \%)$ & 0 & $(0.0 \%)$ & 2 & $(16.7 \%)$ \\
\hline & 2 & 0 & $(0.0 \%)$ & 1 & $(14.3 \%)$ & 0 & $(0.0 \%)$ \\
\hline Patients experiencing DLT & & 0 & $(0.0 \%)$ & 0 & $(0.0 \%)$ & 0 & $(0.0 \%)$ \\
\hline
\end{tabular}

DLT: Dose-limiting toxicity.

follows: i) complete response $(\mathrm{CR})$ : VAS=0 without concomitant increase in analgesic intake; ii) partial response (PR): pain reduction $\geq 2$ points (VAS) without an increase in analgesics intake, or analgesic reduction $\geq 25 \%$ without worsening of pain; iii) pain progression: increase of pain $\geq 2$ VAS points with stable analgesic therapy or as $\geq 25 \%$ analgesic intake with worsened or stable pain; iv) indeterminate response: any other situation. In addition, data on ECOG and QoL were categorized as worse, stable, or improved while comparing follow-up with baseline values.

Ethical consideration. The Institutional Ethic Committee approved this trial (UCSC/CB/RT-2012/3). The study was designed in agreement with the Helsinki Declaration (2008 revision). Before enrolment in the trial, patients had to provide a written informed consent.

\section{Results}

Patient characteristics. Twenty-five patients (median age $=71$ years; range $=58-86$ years) were enrolled in this trial. Median follow-up at the time of the analysis was 7.4 months (range=3-24 months). All patients had PCa with multiple BM localized in bony pelvis, lumbar spine, and femurs. ECOG was 1,2 , and 3 in $56 \%, 20 \%$, and $24 \%$ of patients, respectively. The CTV definition in enrolled patients is shown in Table I.

Maximum tolerated dose. In the 1st dose level, six patients were enrolled and in the 2nd cohort there were seven patients due to an early death ( $<3$ months). In these two cohorts, no DLT was recorded and therefore the study proceeded with enrollment in the 3rd cohort. Even at this level no DLTs were recorded and therefore the MTD was defined as 15 Gy in 4 fractions.

Toxicity. Table II illustrates the acute toxicity rates. The most frequent RT-related toxicity was G1 gastrointestinal toxicity (4 patients, 16\%). Two patients (8\%) experienced G2 gastrointestinal toxicity with nausea requiring antiemetic drugs. Grade 1-2 pain flare-up was observed in three patients (12\%). Overall, no cases of G3 acute toxicity or death within one month from RT were recorded. Finally, two patients experienced G1 late skin toxicity.

Symptom relief and QoL. The palliative response rate (CR plus $\mathrm{PR})$ in the whole patients' population was $76.0 \%(19 / 25$ patients). Fifteen days after MHB-RT, VAS was significantly reduced compared to baseline [post-treatment versus pretreatment: 2.7 and 5.3 , respectively $(p<0.001)]$. The mean VAS reduction was $53.0 \%$. Nine patients $(36.0 \%)$ showed pain CR and 10 patients $(40.0 \%)$ reported PR. Two patients $(8.0 \%)$ experienced pain progression at the 2 months follow-up visit. One of them died three weeks later. Another death was recorded 18 months after diagnosis. Four patients (16.0\%) had an indeterminate response according to International Consensus on Palliative Radiotherapy. During the first control visit after RT, 11 patients (44\%) showed improved ECOG while $11(44 \%)$ had the same value as at baseline. No statistical differences were found between CLAS 1, CLAS 2 and CLAS 3 evaluations before and after treatment.

\section{Discussion}

A dose-escalation trial was planned and performed with the aim to establish the MTD of MHB-RT in patients with PCa $\mathrm{BM}$ using an accelerated regimen of four fractions in two days.

Main limitations of this analysis are the small sample size (due to the study design) and the lack of late toxicity evaluation given the short observation period. Nevertheless, we can estimate that the risk of late toxicity is extremely unlikely considering the low equivalent dose value in terms of conventional fractionation (EQD2: $20.2 \mathrm{~Gy}$ ) of the defined MTD (15 Gy in 4 fractions). 
In a previous study of our group (20) we demonstrated that MHB (12 Gy in two days, $3 \mathrm{~Gy} /$ fraction, 2 fractions/day) is effective in terms of pain relief $(37.5 \%$ CR; $38.1 \%$ PR) and safe (grade 3 toxicity: $1.1 \%$, grade 4 toxicity: $0.0 \%$ ). However, Salazar and colleagues (10) using a 2D-traditional RT technique with anterior-posterior and posterior-anterior opposed fields reported a lower efficacy and higher toxicity rates of this schedule compared to $15 \mathrm{~Gy}$ in 5 daily fractions, in a subgroup of patients with $\mathrm{BM}$ from PCa.

Therefore, we evaluated the feasibility of a regimen combining the advantages of a higher dose (compared to 12 Gy) and a shorter duration (compared to 5 fractions per day). In particular, we hypothesized that the use of a multiple beams conformal technique would allow treatment intensification. Our results confirmed this hypothesis and justified further the evaluation of this regimen in a phase II trial.

More generally, this study confirmed the efficacy and tolerability of accelerated palliative RT delivered in only two days (four fractions), as previously observed in previous studies on different clinical settings (21-27). Furthermore, our analysis is in agreement with several studies showing a high palliative response rate after MHBRT in PCa BM (28-30), particularly using fractionated regimens (30).

In conclusion, based on our preliminary data on safety and palliative response (CR: $36.0 \%$; PR: $40 \%$ ), this regimen could be introduced in clinical practice, particularly due to its short duration in hospitalised patients and in those living far from RT centers.

We can point out that the interest in MHB-RT in this category of patients has gradually decreased in view of the availability of radiopharmaceuticals especially and more recently 223-Radium (31). However, considering the low costs and the good tolerability observed in our analysis, comparative studies between metabolic RT and MHB-RT seem justified.

Finally, considering the availability of new systemic treatments in metastatic castration-resistant $\mathrm{PCa}$, further studies could be designed to define the optimal combination of this regimen with these drugs.

\section{Conflicts of Interest}

The Authors state that they have no conflicts of interest regarding this study.

\section{Authors' Contributions}

Conception and Design: AGM, AZ, MB, SiC and FD; Data Collection: AZ, GM, GS, SaC, and FD; Analysis and Interpretation of Data: AZ, MB, AA, EF and FC; Manuscript Writing AGM, AZ, $\mathrm{MB}$, and SiC. All Authors read and approved the final manuscript and gave consent to publication.

\section{Acknowledgements}

This research did not receive any specific grant from funding agencies in the public, commercial, or not-for-profit sectors.

\section{References}

1 Bray F, Ferlay J, Soerjomataram I, Siegel RL, Torre LA and Jemal A: Global cancer statistics 2018: GLOBOCAN estimates of incidence and mortality worldwide for 36 cancers in 185 countries. CA Cancer J Clin 68: 394-424, 2018. PMID: 30207593. DOI: $10.3322 /$ caac. 21492

2 Cathomas R, Bajory Z, Bouzid M, El Ghoneimy A, Gillessen S, Goncalves F, Kacso G, Kramer G, Milecki P, Pacik D, Tantawy $\mathrm{W}$ and Lesniewski-Kmak K: Management of bone metastases in patients with castration-resistant prostate cancer. Urol Int 92: 377-386, 2014. PMID: 24802278. DOI: 10.1159/000358258

3 James ND, Spears MR, Clarke NW, Dearnaley DP, De Bono JS, Gale J, Hetherington J, Hoskin PJ, Jones RJ, Laing R, Lester JF, McLaren D, Parker CC, Parmar MKB, Ritchie AWS, Russell JM, Strebel RT, Thalmann GN, Mason MD and Sydes MR: Survival with newly diagnosed metastatic prostate cancer in the "docetaxel ra": data from 917 patients in the control arm of the STAMPEDE Trial (MRC PR08, CRUK/06/019. Eur Urol 67: 1028-1038, 2015. PMID: 25301760. DOI: 10.1016/j.eururo. 2014.09.032

4 Drake CG: Visceral Metastases and Prostate Cancer Treatment: 'Die Hard,' 'Tough Neighborhoods,' or 'Evil Humors'? Oncology (Williston Park, N.Y.) 28: 974-980, 2014. PMID: 25403629.

5 Fairchild A and Lutz S: Palliative radiotherapy for bone metastases. In: Decision making in radiation oncology. Brady LW and Lu JJ (eds.). Springer, Berlin, chapter 1B, pp. 25-44, 2011.

6 Lutz S, Spence C, Chow E, Janjan N and Connor S: Survey on use of palliative radiotherapy in hospice care. J Clin Oncol 22: 3581-3586, 2004. PMID: 15337808. DOI: 10.1200/JCO.2004. 11.151

7 Fitzpatrick PJ and Rider WD: Half-body radiotherapy of advanced cancer. Am J Surg 132: 75-79, 1976. PMID: 962003.

8 Salazar OM, Rubin P, Hendrickson FR, Komaki R, Poulter C, Newall J, Asbell SO, Mohiuddin M and Van Ess J: Single-dose half body irradiation for palliation of multiple bone metastases from solid tumors. Final Radiation Therapy Oncology Group report. Cancer 58: 29-36, 1986. PMID: 2423225. DOI: 10.1002/1097-0142(19860701)58:1<29::aid-cncr2820580107> 3.0.co;2-2

9 Salazar OM, DaMotta NW, Bridgman SM, Cardiges NM and Slawson RG: Fractionated half-body irradiation for pain palliation in widely metastatic cancers: comparison with single dose. Int J Radiat Oncol Biol Phys 36: 49-60, 1996. PMID: 8823258. DOI: 10.1016/s0360-3016(96)00248-9

10 Salazar OM, Sandhu T, da Motta NW, Escutia MA, LanzósGonzales E, Mouelle-Sone A, Moscol A, Zaharia M and Zaman S: Fractionated half-body irradiation (HBI) for the rapid palliation of widespread, syntomatic, metastatic bone disease: a randomised phase III trial of the International Atomic Energy Agency (IAEA). Int J Radiat Oncol Biol Phys 50: 765-775, 2001. PMID: 11395246. DOI: 10.1016/s0360-3016(01)01495-x 
11 Arbuck SG: Workshop on phase I study design. Ninth NCI/EORTC New Drug Development Symposium, Amsterdam, March 12, 1996. Ann Oncol 7: 567-573, 1996. PMID: 8879369.

12 Cox JD, Stetz J and Pajak TF: Toxicity criteria of the Radiation Therapy Oncology Group (RTOG) and the European Organization for Research and Treatment of Cancer (EORTC). Int J Radiat Oncol Biol Phys 31: 1341-1346, 1995. PMID: 7713792. DOI: 10.1016/0360-3016(95)00060-C

13 Melzack R: The McGill Pain Questionnaire: major properties and scoring methods. Pain 1: 277-299, 1975. PMID: 1235985.

14 Sutherland HJ, Walker P and Till JE: The development of a method for determining oncology patients' emotional distress using linear analogue scales. Cancer Nurs 11: 303-308, 1988. PMID: 3233581.

15 Bentzen SM, Joiner MC: The linear-quadratic approach in clinical practice. In: Joiner MC, AJ Van der Koger (ed.). Basic Clinical Radiobiology, 5th Edition. CRC Press/Taylor \& Francis Group, Boca Raton, FL pp. 111-123, 2018.

16 Landberg T, Chavaudra J, Dobbs J, Gerard JP, Hanks G, Horiot JC, Johansson KA, Möller T, Purdy J, Suntharalingam N and Svensson H: ICRU Report 62: prescribing, recording and reporting photon beam therapy (supplement to ICRU Report 50). J ICRU 32(1): 48-51, 1999. DOI: 10.1093/jicru/os32.1.48

17 Morganti AG, Deodato F, Zizzari S, Cilla S, Digesu' C, Macchia G, Panunzi S, De Gaetano A, Piermattei A, Cellini N and Valentini V: Complexity index (COMIX) and not type of treatment predicts undetected errors in radiotherapy planning and delivery. Radioter Oncol 89: 320-329, 2008. PMID: 18701176. DOI: 10.1016/j.radonc.2008.07.009

18 Deodato F, Cilla S, Massaccesi M, Macchia G, Ippolito E, Caravatta L, Picardi V, Romanella M, Di Falco C, Bartollino A, Valentini V, Cellini N, De Spirito M, Piermattei A and Morganti AG: Daily on-line set-up correction in 3D-conformal radiotherapy: is it feasible? Tumori 98: 441-444, 2012. PMID: 23052159. DOI: $10.1700 / 1146.12637$

19 Chow E, Hoskin P, Mitera G, Zeng L, Lutz S, Roos D, Hahn C, van der Linden Y, Hartsell W, Kumar E and International Bone Metastases Consensus Working Party: Update of the international consensus on palliative radiotherapy endpoints for future clinical trials in bone metastases. Int J Radiat Oncol Biol Phys 82: 17301737, 2012. PMID: 21489705. DOI: 10.1016/ j.jijrobp.2011.02.008

20 Macchia G, Ferro M, Cilla S, Buwenge M, Ianiro A, Boccardi M, Picardi V, Ferro M, Arena E, Zamagni A, Cammelli S, Valentini V, Morganti AG and Deodato F: Efficacy and safety of 3D-conformal half body irradiation in patients with multiple bone metastases. Clin Exp Metastasis 35: 747-752, 2018. PMID: 30251079. DOI: 10.1007/s10585-018-9939-1

21 Farina E, Macchia G, Buwenge M, Siepe G, Zamagni A, Cammelli S, Cilla S, Wondemagegnhu T, Woldemariam AA, Uddin AFMK, Sumon MA, Cellini F, Deodato F and Morganti AG: Radiotherapy in palliation of thoracic tumors: a phase I-II study (SHARON project). Clin Exp Metastasis 35: 739-746, 2018. PMID: 30298381. DOI: 10.1007/s10585-018-9942-6

22 Capuccini J, Macchia G, Farina E, Buwenge M, Genovesi D, Caravatta L, Nguyen NP, Cammelli S, Cilla S, Wondemagegnhu T, Uddin AFMK, Aziz Sumon M, Cellini F, Valentini V, Deodato $\mathrm{F}$ and Morganti AG: Short-course regimen of palliative radiotherapy in complicated bone metastases: a phase I-II study (SHARON Project). Clin Exp Metastasis 35: 605-611, 2018. PMID: 30121938. DOI: 10.1007/s10585-018-9931-9
23 Caravatta L, Deodato F, Ferro M, Macchia G, Massaccesi M, Cilla S, Padula GD, Mignogna S, Tambaro R, Carrozza F, Flocco M, Cantore G, Scapati A, Buwenge M, Sticca G, Valentini V, Cellini $\mathrm{N}$ and Morganti AG: A phase I study of short-course accelerated whole brain radiation therapy for multiple brain metastases. Int $\mathrm{J}$ Radiat Oncol Biol Phys 84: e463-468, 2012. PMID: 22909415. DOI: $10.1016 /$ j.jirobp. 2012.06.023

24 Caravatta L, Deodato F, Ferro M, Macchia G, Massaccesi M, Cilla S, Tambaro R, Mignogna S, Padula GD, Musacchio M, Flocco M, Cantore G, Scapati A, Bogale S, Balducci M, Valentini V, Cellini N and Morganti AG: Results of a phase II study of short-course accelerated radiation therapy (SHARON) for multiple brain metastases. Am J Clin Oncol 38: 395-400, 2015. PMID: 26214084. DOI: 10.1097/COC.0b013e3182a0e826

25 Farina E, Capuccini J, Macchia G, Caravatta L, Nguyen NP, Cammelli S, Farioli A, Zanirato Rambaldi G, Cilla S, Wondemagegnhu T, Uddin AFMK, Sumon MA, Genovesi D, Buwenge M, Cellini F, Valentini V, Deodato F and Morganti AG: Phase I-II study of short-course accelerated radiotherapy (SHARON) for palliation in head and neck cancer. Anticancer Res 38: 2409-2414, 2018. PMID: 29599369. DOI: 10.21873/ anticanres.12491

26 Farina E, Capuccini J, Macchia G, Caravatta L, Nguyen NP, Cammelli S, Zanirato Rambaldi G, Cilla S, Wondemagegnhu T, Uddin AFMK, Sumon MA, Genovesi D, Buwenge M, Cellini F, Valentini V, Deodato $F$ and Morganti AG: Short course accelerated radiation therapy (SHARON) in palliative treatment of advanced solid cancer in older patients: a pooled analysis. J Geriatr Oncol 9: 359-361, 2018. PMID: 29398455. DOI: 10.1016/j.jgo.2018.01.004

27 Caravatta L, Padula GD, Macchia G, Ferrandina G, Bonomo P, Deodato F, Massaccesi M, Mignogna S, Tambaro R, Rossi M, Flocco M, Scapati A, Scambia G, Pacelli F, Valentini V, Cellini $\mathrm{N}$ and Morganti AG: Short course accelerated radiation therapy (SHARON) in palliative treatment of advanced pelvic malignancies: a phase I study. Int J Radiat Oncol Biol Phys 83: e627-631, 2012. PMID: 22580117. DOI: 10.1016/j.ijrobp. 2011.10.081

28 Kuban DA, Delbridge T, el-Mahdi AM and Schellhammer PF: Half-body irradiation for treatment of widely metastatic adenocarcinoma of the prostate. J Urol 141: 572-574, 1989. PMID: 2465419. DOI: 10.1016/s0022-5347(17)40897-4

29 Rowland CG, Bullimore JA, Smith PJ and Roberts JB: Halfbody irradiation in the treatment of metastatic prostatic carcinoma. Br J Urol 53: 628-629, 1981. PMID: 6172173.

30 Zelefsky MJ, Scher HI, Forman JD, Linares LA, Curley T and Fuks Z: Palliative hemiskeletal irradiation for widespread metastatic prostate cancer: a comparison of single dose and fractionated regimens. Int J Radiat Oncol Biol Phys 17: 12811285, 1989. PMID: 2480941. DOI: 10.1016/0360-3016(89) 90537-3

31 Shirley M and McCormack PL: Radium-223 dichloride: a review of its use in patients with castration-resistant prostate cancer with symptomatic bone metastases. Drugs 74: 579-586, 2014. PMID: 24610703. DOI: 10.1007/s40265-014-0198-4

Received July 1, 2019

Revised July 17, 2019

Accepted July 18, 2019 